\title{
A fine time for contractual alterations
}

\section{Andrew Huxley}

IT has long been supposed that the elementary event which produces tension in a muscle fibre, or causes it to shorten, may be a change in the orientation of the head of a myosin molecule; the head spans the gap between a thick (myosin) and a thin (actin) filament, and acts as a crank pulling the thin filament into the array of thick filaments which forms the A band of the striation pattern $^{1,2}$. Such an event would alter the distribution of mass projected onto the fibre axis. In their report on page 156 of this issue ${ }^{3}$, describing experiments with low-angle X-ray diffraction, Irving et al. show that the expected change does indeed accompany the 'working stroke' of the force generators.

\section{Reflection}

A feature of the low-angle X-ray diffraction pattern of striated muscle is a reflection on the meridian at a position corresponding to an axial spacing of $14.5 \mathrm{~nm}$, the interval along each thick filament between successive 'crowns' where the heads of myosin molecules project from the backbone of the filament. Nearly a decade ago, H. E. Huxley and his colleagues $^{4}$ showed that the intensity of this spot fell to about half when an active muscle shortened quickly by $0.5-1$ per cent of its length, and recovered with a time constant of about $10 \mathrm{~ms}$ (frog muscle at $0^{\circ} \mathrm{C}$ ). The time resolution of their measurements (X-ray quanta counted in $1 \mathrm{~ms}$ bins and shortening complete in about $1 \mathrm{~ms}$ ) was impressive at the time. But it was not good enough to show clearly whether the drop in intensity of the $14.5 \mathrm{~nm}$ meridional reflection was an accompaniment of the shortening itself or of the redevelopment of tension that occurs in the subsequent 1-2 ms, and represents (in at least one sense) the working stroke of the crossbridges $^{5,6}$. Irving et al. have taken advantage of the improved time resolution ( $0.2 \mathrm{~ms}$ bins) now available at the SERC synchrotron facility at Daresbury to repeat the experiment. They show decisively that the change does not begin until the shortening is complete and that it develops with a time course similar to that of the tension redevelopment (that is, of the working stroke).

In addition to its theoretical significance, the experiment of Irving et al. was a technical tour de force. The tissue used was a single muscle fibre (from a frog); the step change in length was complete in $0.12 \mathrm{~ms}$; and the length change in the segment of the fibre traversed by the
X-ray beam was recorded continuously with an optical device ${ }^{7}$ which monitors the movement of the striations at each end of the segment with time resolution of a few microseconds.

Irving et al. show, by computer modelling, that the intensity change can be well explained by tilting of the myosin heads from an attitude roughly perpendicular to the fibre axis with time course and extent expected from a kinetic scheme previously devised to fit the mechanical responses to step changes of length ${ }^{8}$. They admit, however, that this is not the only type of crossbridge movement that might be causing the $\mathrm{X}$-ray change; it might, for example, be a sliding movement of some of the heads along the thin filament, or it might be attachment at intermediate positions of myosin heads that were not attached to actin before the step. But the observation does appear to be an unambiguous demonstration that the working stroke is accompanied by a redistribution along the fibre axis of the material composing the crossbridges (the heads of myosin molecules).

The experiment is also relevant to the interpretation of a phenomenon reported a few months ago by Lombardi $e t$ al. ${ }^{9}$, one of the groups responsible for the new X-ray results. It was already known ${ }^{6}$ that when two step releases are applied within 1-2 ms, the subsequent time course of tension is much the same as that following a single release with amplitude equal to their sum. Lombardi et al. showed however that when the interval is increased to $10-15 \mathrm{~ms}$, the second release is followed by a quick redevelopment of tension almost as great as if the first release had not taken place. The time in which this 'repriming' of the ability to perform a working stroke occurs is much too short for one to be able to assume that crossbridges have completed their biochemical cycle using one molecule of ATP, which implies that the muscle fibre can perform several working strokes with the energy provided by the hydrolysis of a single molecule of ATP. The time course of the repriming is similar to that of the recovery of intensity of the $14.5 \mathrm{~nm}$ meridional reflection that was seen both by H. E. Huxley et al. ${ }^{4}$ and by Irving et $a l^{3}$, suggesting that the crossbridges have returned to their previous attitude.

Both the repriming and the recovery of the $14.5 \mathrm{~nm}$ intensity, with the observed time course, are predicted by the same kinetic scheme ${ }^{8}$ that accounted for the initial drop of intensity. The relevant feature of this scheme is that a crossbridge which has not completed all the steps of its working stroke, and which is put into compression by the imposed quick shortening, is able to detach with a rate constant corresponding to the speed of repriming. It can then rapidly reattach further along the actin filament in the attitude corresponding to the start of a fresh working stroke.

\section{Drawback}

The drawback of the kinetic scheme as it stands is that it does not set a limit to the number of times that a partial working stroke can be performed with the utilization of one molecule of ATP; in its present form it is therefore thermodynamically inconsistent. One hopes however that a modification to the scheme (for example, perhaps postulating that the crossbridge does not detach but is brought back to its initial attitude by a shortening of the S-2 segment of the myosin molecule driven by the phosphate-release step of the ATPase cycle) may make it satisfactory in that respect without losing its relevant kinetic features.

There have been other suggestions that each crossbridge may perform several working strokes for the use of one molecule of ATP (see, for example, refs $10-12$ ). These are still controversial, and not all of them assume the same timescale as the experiment of Lombardi et al. ${ }^{9}$. This is a lively field of research at present, with several groups studying the mechanical behaviour of isolated filaments in preparations where the force contributed by individual molecules can be detected either as noise or as individual impulses. Another technique now in use is quick freezing for electron microscopy; this is probably the only approach that can decide whether the structural alteration in a crossbridge really is a change of its angle relative to the fibre axis.

Sir Andrew Huxley is a Fellow of Trinity College, Cambridge CB2 1TQ, UK.

1. Reedy. M. K., Holmes, K. C. \& Tregear, R. T. Nature 207, 1276-1280 (1965)

2. Huxley, H. E. Science 164, 1356-1366 (1969)

3. Irving, M., Lombardi, V., Piazzesi, G. \& Ferenczi, M. Nature 357, 156-158 (1992)

4. Huxley, H. E. et al. J. molec. Biol. 169, 469-506 (1983).

5. Huxley, A. F. \& Simmons, R. M. Nature 233, 533-538 (1971)

6. Ford, L. E., Huxley, A. F. \& Simmons, R. M. J. Physiol., Lond. 269, 441-515 (1977).

7. Huxley, A. F., Lombardi, V. \& Peachey, L. D. J. Physiol, Lond. 317, 12-13P (1981).

8. Piazzesi, G. Francini, F., Linari, M. \& Lombardi, V. J. Physiol., Lond. 445, 659-711 (1992).

. Lombardi, V., Piazzesi, G. \& Linari, M. Nature 355 638-641 (1992)

10. Yanagida, T., Arata, T. \& Oosawa, F. Nature 316 366-369 (1985)

1. Ishijima, A., Doi, T., Sakurada, K. \& Yanagida, T. Nature 352, 301-306 (1991)

12. Brenner, B. Proc. natn. Acad. Sci. U.S.A. 88, 10490 10494 (1991). 